\title{
Effect of Caffeine on Otoacoustic Emissions
}

\author{
${ }^{1}$ Department of Audiology and Speech-Language Pathology, Nitte \\ Institute of Speech and Hearing, Mangalore, Karnataka, India \\ 2Department of Audiology and Speech-Language Pathology, \\ Yenepoya University, Mangalore, Karnataka, India \\ ${ }^{3}$ Department of Audiology and Speech-Language Pathology, Madras \\ ENT Research Foundation (P) Ltd, Chennai, Tamil Nadu, India
}

Drepath Sanish Kumar ${ }^{1}$ Athira Rajan ${ }^{1}$ Srikanth Nayak ${ }^{1,2} \quad$ Arya Vijay $^{1}$ Sathish Kumar ${ }^{3}$

\author{
Address for correspondence Sathish Sanish Kumar, MSc, \\ Department of Audiology and Speech-Language Pathology, Madras \\ ENT Research Foundation (P) Ltd, Chennai, 600028, India \\ (e-mail: sathish.sreeni58@gmail.com).
}

\begin{abstract}
Keywords

- caffeine

- coffee

- cochlea

- otoacoustic emissions

Introduction Caffeine is consumed everyday in a variety of ways by a large population around the world, including coffee, tea, and soft drinks. Therefore, there is a significant need to explore the effects of caffeine on human auditory system. The present study aimed to investigate the effect of caffeine on cochlea using distortion product otoacoustic emission (DPOAE).

Materials and Methods Thirty-three young adults were recruited for the study. The effect of caffeine on cochlea was assessed using DPOAE at frequencies between 1 to $10 \mathrm{kHz}$ before and after caffeine consumption.

Results The signal to noise ratio of DPOAE was compared before and after coffee intake, which revealed no significant differences.

Conclusion The current study results suggest that caffeine has no effect on cochlear functioning.
\end{abstract}

\section{Introduction}

Coffee is a nonalcoholic beverage which is used most widely in industrialized countries. Coffee is a mixture of many components in which caffeine is the active constituent. ${ }^{1}$ There is approximately $60 \pm 120 \mathrm{mg}$ of caffeine in $150 \mathrm{~mL}$ of coffee. ${ }^{2}$ Caffeine reduces the cerebral blood flow inducing relative brain hypoperfusion, but at the same time, it increases energy metabolism throughout the brain. ${ }^{3}$ It has been shown that a 250-mg dose of caffeine reduces resting cerebral blood flow by $22 \%$ and middle cerebral artery blood velocity by $13 \%{ }^{4}$ Coffee contains many bioactive substances which result in physiological effects with functions including anti-inflammatory, antioxidant, antifibrotic, and anticancer effects. ${ }^{5}$ Regular coffee intake leads to the risks of cancer, diabetes, cardiovascular disease, Parkinson's disease, liver disease, dementia, and depression and can even increase the heart rate and ambulatory blood pressure. ${ }^{6}$

According to the Global Burden of Disease, hearing loss is a major health issue and over $5 \%$ of the world population experience hearing loss, leading to communication difficulties and social withdrawal. ${ }^{7}$ Few studies investigated the long-term effects of caffeine consumption using pure tone audiometry ${ }^{8}$ and speech in noise test ${ }^{9}$ and found a low incidence of hearing loss. On the other hand, caffeine's short-term effect on the auditory system in humans was less researched. Thus, the present study investigates the short-term effect of coffee on the cochlea's outer hair cell $(\mathrm{OHC})$ functioning using the distortion product otoacoustic emissions (DPOAEs) test.
Published online

September 10, 2021
DOI https://doi.org/ 10.1055/s-0041-1735385 ISSN 2581-9607
(C) 2021. Indian Society of Otology.

This is an open access article published by Thieme under the terms of the Creative Commons Attribution-NonDerivative-NonCommercial-License, permitting copying and reproduction so long as the original work is given appropriate credit. Contents may not be used for commercial purposes, or adapted, remixed, transformed or built upon. (https://creativecommons.org/licenses/by-nc-nd/4.0/).

Thieme Medical and Scientific Publishers Pvt. Ltd. A-12, 2nd Floor, Sector 2, Noida-201301 UP, India 


\section{Method}

Thirty young normal-hearing adults (age range of 18-23 years) were included in the present study (20 males and 10 females). Institute ethical committee clearance was taken for the study. The volunteer participants were informed about the study's aim, and written consents were taken before participating in the study.

All the participants had the habit of consuming coffee over more than 10 years with a minimum of $100 \mathrm{~mL}$ of coffee twice a day, and participants who had DPOAEs with $6 \mathrm{~dB}$ signal-to-noise ratio (SNR) for frequencies between 1,000 and $10,000 \mathrm{~Hz}$ were included for the study. Any individual with middle ear pathology, any medical ear history, the habit of smoking, alcohol consumption, and hearing music with headphone for more than 2 hours per day was excluded from the study. All the participants had pure-tone thresholds $\leq 15 \mathrm{~dB}$ hearing level at octave frequencies from 250 to $8,000 \mathrm{~Hz}$ for air conduction and 250 to $4,000 \mathrm{~Hz}$ for bone conduction and "A" type tympanogram with acoustic reflexes present in ipsilateral and contralateral conditions for 500, 1,000 , and $2,000 \mathrm{~Hz}$. All the participants were instructed to avoid the intake of coffee or any caffeine products and avoid any noise exposure, including listening to music from the personal music system before 12 hours of participation.

DPOAE testing was performed using Otodynamics ILO-V6 software. The subjects were made to sit comfortably and instructed not to move during the test. The probe was inserted gently into the ear canal with an appropriate probe tip and was ensured to have a good fit and not removed until the test's completion. A ratio of 1.22 between $\mathrm{f} 1$ and $\mathrm{f} 2$ was used to elicit the DPOAEs. The intensity level of $\mathrm{f} 1$ and $\mathrm{f} 2$ were 65 and $55 \mathrm{~dB}$ sound pressure level, respectively. Emissions were measured at eight frequency points. The SNR at various 2f1-f2 of frequency was noted down. Initially, baseline DPOAEs were taken from both ears simultaneously. Then, these subjects were introduced with $100 \mathrm{~mL}$ of coffee which was customized for the study. DPOAEs were done simultaneously in both ears after a break of 15 minutes.

The data obtained were subjected to statistical analysis to compare the SNRs of DPOAEs pre- and post coffee intake. Descriptive statistics and Wilcoxon signed-rank test were done to find the mean and standard deviations and statistical significance of the SNR response of DPOAEs pre- and post coffee intake.

\section{Results}

The mean and standard deviation of DPOAE's SNR, pre- and post coffee intake is shown in - Table 1. In general, when compared with the baseline DPOAEs, the mean SNR value of overall DPOAEs was slightly reduced in both ears post the intake of coffee. Wilcoxon signed-rank test was done to find the difference between pre- and post the intake of coffee. Results showed no statistically significant difference in the SNRs of DPOAEs in both ears for pre- and post coffee intake.

\section{Discussion}

The present study aimed to examine the short-term effect of coffee on OHC functioning, measured using DPOAEs test. Our results revealed no significant difference between baseline and post the intake of coffee DPOAEs at all frequencies. Numerous studies reported caffeine to enhance cortical arousal and perceptual sensitivity in the auditory domain. ${ }^{10}$ In support of this mechanism, the electrophysiological studies shred the evidence of enhanced amplitude and reduced latencies in peripheral and central auditory responses. ${ }^{11-14}$ The changes in neural responses are attributed to the increased release of neurotransmitters in the auditory neural pathway. However, the effect of caffeine at the cochlear level is unexplored.

The caffeine effect on the cochlea in animals has been reported in the literature. The introduction of caffeine in the guinea pigs measured using membrane potentials results in depolarization of $\mathrm{OHCs}$ by closing $\mathrm{K}^{+}$channels, ${ }^{15}$ reduces the movement of OHCs, and suppresses the cochlear amplifier. ${ }^{16,17}$ On the other hand, Bobbin ${ }^{18}$ studied the effect of caffeine with different doses $(1,3$, and $10 \mathrm{mM})$ on the peripheral auditory system using high-frequency DPOAE (8 and $12 \mathrm{kHz}$ ) and cochlear and auditory nerve potentials (compound action potentials [CAP], summating potential [SP], and cochlear microphonics $[\mathrm{CM}])$ at various signal intensities. He found that caffeine suppresses the CAP and SP responses, increased N1 latency, suppression in DPOAE responses only at low intensities, and increased $\mathrm{CM}$ responses for $1 \mathrm{kHz}$

Table 1 Mean and standard deviation of DPOAEs pre- and post the intake of coffee

\begin{tabular}{|l|l|l|l|l|}
\hline \multirow{2}{*}{$\begin{array}{l}\text { Frequencies } \\
(\mathrm{kHz})\end{array}$} & \multicolumn{2}{|c|}{ Right ear } & \multicolumn{2}{c|}{ Left ear } \\
\cline { 2 - 5 } & Pre & Post & Pre & Post \\
\hline 1 & $5.67(1.71)$ & $5.57(1.73)$ & $5.39(1.62)$ & $6.64(1.75)$ \\
\hline 1.4 & $15.16(1.69)$ & $13.8(1.5)$ & $14.05(2.03)$ & $14.1(1.94)$ \\
\hline 2 & $14.76(1.58)$ & $13.02(1.42)$ & $12.76(1.97)$ & $12.63(1.93)$ \\
\hline 2.4 & $10.49(1.96)$ & $9.82(1.53)$ & $9.35(1.94)$ & $9.31(1.86)$ \\
\hline 4 & $13.37(1.51)$ & $12.67(1.37)$ & $14.6(1.4)$ & $13.08(1.67)$ \\
\hline 6 & $17.99(1.77)$ & $15.63(1.61)$ & $18.25(1.29)$ & $18.06(1.42)$ \\
\hline 8 & $8.88(2.09)$ & $7.78(1.8)$ & $7.87(2.49)$ & $7.47(2.34)$ \\
\hline 10 & $7.13(1.95)$ & $5.54(1.73)$ & $3.9(2.09)$ & $3.81(2.15)$ \\
\hline
\end{tabular}

Abbreviation: DPOAE, distortion product otoacoustic emission. 
tone pips but no changes for $10 \mathrm{kHz}$ tone pips. In general, caffeine shows strong differences in the peripheral auditory responses only at low intensities compared with high intensities. The substantial response changes observed for low intensities can be attributed to the disruption in the $\mathrm{OHC}$ function (cochlear amplification) caused by the caffeine.

To our knowledge, no studies had explored cochlear function as the effect of caffeine in humans. The result found in our study must be considered with precaution at this point due to various limitations. First, the animal studies mentioned above are difficult to consider to answer our findings. Since the studies discussed above have caffeine concentration in millimolar units, such concentrations cannot be achieved in everyday coffee consumptions. Second, the effect of caffeine is dose-dependent and varies between the types of coffee (brewed, instant, or canned). ${ }^{8}$ Third, since the caffeine effect is dose-dependent, we do not know caffeine administered to our subjects enough to stimulate the auditory system. Since the electrophysiological changes caused by caffeine are well known, they can be used to determine an appropriate caffeine dose. So, future studies are recommended to evaluate cochlear response along with electrophysiological responses with careful consideration of dose and type of coffee.

\section{Conflict of Interest}

The authors declare no potential conflict of interest.

\section{Acknowledgments}

We thank the Department of Audiology and Speech Language Pathology, Nitte Institute of Speech and Hearing, Mangalore, for permitting us to conduct the study.

\section{References}

1 Ruxton CHS. The impact of caffeine on mood, cognitive function, performance and hydration: a review of benefits and risks. Nutr Bull 2008;33(1):15-25

2 Barone JJ, Roberts HR, Caffeine consumption. Food Chem Toxicol 1996;34(1):119-129

3 Nehlig A, Daval JL, Debry G, Caffeine and the central nervous system: mechanisms of action, biochemical, metabolic and psychostimulant effects. Brain Res Rev 1992;17(2):139-170

4 Lunt MJ, Ragab S, Birch AA, Schley D, Jenkinson DF. Comparison of caffeine-induced changes in cerebral blood flow and middle cerebral artery blood velocity shows that caffeine reduces middle cerebral artery diameter. Physiol Meas 2004;25(2):467-474

5 Gaascht F, Dicato M, Diederich M. Coffee provides a natural multitarget pharmacopeia against the hallmarks of cancer. Genes Nutr 2015;10(6):51

6 Green PJ, Suls J. The effects of caffeine on ambulatory blood pressure, heart rate, and mood in coffee drinkers. J Behav Med 1996;19(2):111-128

7 Chadha S, Cieza A, Promoting global action on hearing loss: world hearing day. Intl J Audiol 2017;56(3):145-147

8 Lee SY, Jung G, Jang MJ, et al. Association of coffee consumption with hearing and tinnitus based on a national population-based survey. Nutrients 2018;10(10):E1429

9 Machado-Fragua MD, Struijk EA, Yévenes-Briones $\mathrm{H}$, Caballero FF, Rodríguez-Artalejo F, Lopez-Garcia E. Coffee consumption and risk of hearing impairment in men and women. Clin Nutr ;40(5):3429-3435

10 Ghahraman MA, Farahani S, Tavanai E, A comprehensive review of the effects of caffeine on the auditory and vestibular systems. Nutr Neurosci 2021;1-1410.1080/1028415X.2021.1918984

11 Dixit A, Vaney N, Tandon OP. Effect of caffeine on central auditory pathways: an evoked potential study. Hear Res 2006;220(1-2):61-66

12 Hong BN, Yi TH, Park R, Kim SY, Kang TH. Coffee improves auditory neuropathy in diabetic mice. Neurosci Lett 2008;441(3):302-306

13 Kawamura N, Maeda H, Nakamura J, Morita K, Nakazawa Y. Effects of caffeine on event-related potentials: comparison of oddball with single-tone paradigms. Psychiatry Clin Neurosci 1996;50(4):217-221

14 Tavanai E, Farahani S, Ghahraman MA, Soleimanian S, Jalaie S. Effects of caffeine on auditory- and vestibular-evoked potentials in healthy individuals: a double-blind placebo-controlled study. J Audiol Otol 2020;24(1):10-16

15 Yamamoto T, Kakehata S, Yamada T, Saito T, Saito $H$, Akaike N. Caffeine rapidly decreases potassium conductance of dissociated outer hair cells of guinea-pig cochlea. Brain Res 1995;677(1):89-96

16 Skellett RA, Crist JR, Fallon M, Bobbin RP. Caffeine-induced shortening of isolated outer hair cells: an osmotic mechanism of action. Hear Res 1995;87(1-2):41-48

17 Slepecky N, Ulfendahl M, Flock A. Effects of caffeine and tetracaine on outer hair cell shortening suggest intracellular calcium involvement. Hear Res 1988;32(1):11-21

18 Bobbin RP. Caffeine and ryanodine demonstrate a role for the ryanodine receptor in the organ of Corti. Hear Res 2002;174(1-2):172-182 